\title{
Eficácia da implementaçáo do cuidado centrado na funcionalidade no declínio funcional: um estu- do quase-experimental
}

Effectiveness of the implementation of function-focused care in functional decline: a quasi-experimental study

Eficacia de la Implementación de los cuidados centrados en la funcionalidad en el deterioro funcional: un estudio cuasiexperimental

Joăo Paulo de Almeida Tavares ${ }^{1}$

(D) https://orcid.org/0000-0003-3027-7978 Joana Grácio ${ }^{2}$

(D) https://orcid.org/0000-0002-0882-2717 Lisa Veiga Nunes ${ }^{3}$

(iD https://orcid.org/0000-0001-9701-027X

${ }^{1}$ Escola Superior de Saúde da Universidade de Aveiro, Aveiro, Portugal

${ }^{2}$ Centro Hospitalar e Universitário de Coimbra, Cirurgia Maxilo-Facial e Plástica, Coimbra, Portugal

${ }^{3}$ Centro Hospitalar e Universitário de Coimbra, Centro de Responsabilidade Integrado de Psiquiatria, Coimbra, Portugal
Autor de correspondência: João Paulo de Almeida Tavares Email: joaoptavares@ua.pt

Recebido: 17.01 .20

Aceite: 18.05.20

\section{Resumo}

Enquadramento: $\mathrm{O}$ cuidado centrado na funcionalidade (CCF) demonstra resultados positivos na prevenção do declínio funcional (DF) das pessoas idosas hospitalizadas (PIH).

Objetivo: Avaliar a eficácia da implementaçáo do CCF em contexto hospitalar na promoção da funcionalidade pelos enfermeiros e na prevenção do DF.

Metodologia: Estudo prospetivo quase-experimental com $101 \mathrm{PIH}$ e 94 enfermeiros de 4 enfermarias. No grupo experimental foi implementado o CCF. As medidas avaliadas foram o CCF e DF em 3 intervalos: baseline-alta (t0), alta-follow-up (t1) e baseline-follow-up ( $\mathrm{t} 2)$.

Resultados: A promoção do CCF foi de $0,46 \pm 0,22$. Verificaram-se diferenças estatisticamente significativas no CCF entre os grupos (experimental e controlo; $p<0,01 ; 0,52 \pm 0,24$ e $0,39 \pm 0,19$, respetivamente). Não houve diferença estatística entre os grupos quanto ao DF em t0 $(p=0,15)$, t 1 $(p=0,15)$ ou t $2(p=0,44)$.

Conclusão: A prática do CCF foi baixa $(46 \%)$, o que pode explicar a falta de impacto na prevenção do DF. Contudo, no grupo experimental, foram desenvolvidas mais atividades de CCF, o que sugere um impacto positivo da implementação desta filosofia.

Palavras-chave: enfermagem; funcionalidade; idoso; declínio funcional; hospitalização

\section{Abstract}

Background: Function-focused care (FFC) has shown positive results in preventing functional decline (FD) among hospitalized older adults.

Objective: To assess the effectiveness of nurses' implementation of FFC in promoting function and preventing FD among hospitalized older adults.

Methodology: Prospective quasi-experimental study with 101 hospitalized older adults and 94 nurses from 4 wards. FFC was implemented in the experimental group. FFC and FD were assessed at 3 intervals: baseline-discharge (t0), discharge-follow up (t1), and baseline-follow-up (t2).

Results: The promotion of FFC was $0.46 \pm 0.22$. Statistically significant differences were found in FFC between groups (experimental and control; $p<0.01 ; 0.52 \pm 0.24$ and $0.39 \pm 0.19$, respectively). No statistically significant difference was found between the groups in FD in t0 $(p=0.15), \mathrm{t} 1(p=$ $0.15)$, and $\mathrm{t} 2(p=0.44)$.

Conclusion: The implementation of FFC was low (46\%), which may explain the lack of impact on FD prevention. However, more FFC activities were developed in the experimental group, which suggests a positive impact of the implementation of this philosophy.

Keywords: nursing; functionality; aged; functional decline; hospitalization

\section{Resumen}

Marco contextual: Los cuidados centrados en la funcionalidad (CCF) muestran resultados positivos en la prevención del deterioro funcional (DF) de las personas mayores hospitalizadas (pessoas idosas hospitalizadas - PIH).

Objetivo: Evaluar la eficacia de la aplicación de CCF en un entorno hospitalario para promover la funcionalidad por los enfermeros y para prevenir el DF.

Metodología: Estudio cuasiexperimental prospectivo con $101 \mathrm{PIH}$ y 94 enfermeros de 4 enfermerías. En el grupo experimental se implementó el CCF. Las medidas evaluadas fueron CCF y DF en 3 intervalos: punto de partida-alta (t0), alta-seguimiento ( $\mathrm{t} 1$ ) y punto de partida-seguimiento $(\mathrm{t} 2)$.

Resultados: La promoción de CCF fue de 0,46 $\pm 0,22$. Hubo diferencias estadísticamente significativas en los CCF entre los grupos (experimental y de control; $p<0,01 ; 0,52 \pm 0,24$ y $0,39 \pm 0,19$, respectivamente). No hubo diferencia estadística entre los grupos en cuanto al DF en t0 $(p=0,15)$, t $1(p=0,15)$ o t $2(p=0,44)$.

Conclusión: La práctica de CCF fue baja (46\%), lo que puede explicar la falta de impacto en la prevención del DF. Sin embargo, en el grupo experimental se desarrollaron más actividades de CCF, lo que sugiere un impacto positivo de la aplicación de esta filosofía.

Palabras clave: enfermería; funcionalidad; anciano; hospitalización

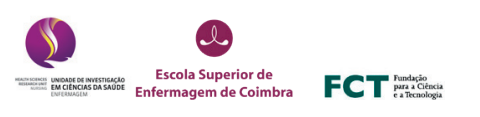

Como citar este artigo: Tavares, J. P. A., Grácio, J., \& Nunes, L. V. (2020). Eficácia da implementação do cuidado centrado na funcionalidade no declínio funcional: um estudo quase-experimental. Revista de Enfermagem Referência, 5(2), e20012. doi:10.12707/RV20012 


\section{Introdução}

A transição demográfica, acompanhada por uma transição epidemiológica e social, implica uma maior demanda de cuidados de saúde, sendo expectável que o número de hospitalizaçóes de pessoas com 65 ou mais anos aumente consideravelmente. Contudo, a hospitalização representa um evento de stress para as pessoas idosas (PI) e está associada a eventos adversos, nomeadamente o declínio funcional (DF; Admi, Shadmi, Baruch, \& Zisberg, 2015). O DF caracteriza-se pela incapacidade para realizar atividade de vida diária, levando à dependência. Em Portugal, verificou-se que o DF é significativo durante e após o internamento hospitalar já que $41,6 \%$ das pessoas idosas hospitalizadas (PIH) não recuperaram o status funcional prévio ao internamento hospitalar (Tavares, Grácio, \& Nunes, 2018). Um dos principais fatores associados ao DF é o baixo nível de atividade física e de estimulação funcional (Zisberg, Shadmi, Gur-Yaish, Tonkikh, \& Sinoff, 2015) das PIH, estimando-se que pelo menos $80 \%$ do tempo é passado na cama (Brown, Redden, Flood, \& Allman, 2009). O cuidado providenciado pelos profissionais de saúde, em especial pelos enfermeiros, pode contribuir para limitar a atividade física (AF)/funcionalidade. Apesar dos benefícios da promoção da $\mathrm{AF} /$ funcionalidade das PIH, este não é o foco do cuidado de enfermagem durante a hospitalizaçáo (Dahlke, Hunter, \& Negrin, 2019; Dermody \& Kovach, 2018; Resnick \& Boltz, 2019). Têm sido implementadas abordagens inovadoras para prevenir o DF como o cuidado centrado na funcionalidade (CCF), que enfatiza o cuidado de enfermagem como essencial para a prevenção do DF (Boltz, Resnick, Capezuti, Shuluk, \& Secic, 2012). Os estudos sobre a implementaçáo do CCF têm demonstrado um aumento da $\mathrm{AF} /$ funcionalidade das PIH e a prevenção do DF e de quedas (Resnick \& Boltz, 2019). Deste modo, o objetivo deste estudo foi avaliar a eficácia da implementação da filosofia do CCF em contexto hospitalar na promoçáo da funcionalidade pelos enfermeiros e na prevenção do DF das $\mathrm{PIH}$.

\section{Enquadramento}

O CCF é uma filosofia emergente do cuidado, que tem sido desenvolvida por Resnick e colaboradores na última década (Resnick \& Boltz, 2019). Trata-se de uma abordagem de cuidado na qual os enfermeiros ajudam as $\mathrm{PIH}$ a envolverem-se nas atividades de vida diária e AF com o objetivo de prevenir o DF evitável. É uma alternativa ao cuidado habitual, na qual o cuidado de enfermagem é desenvolvido com a pessoa idosa e não para esta (Burket, Hippensteel, Penrod, \& Resnick, 2013; Resnick \& Boltz, 2019). O desenvolvimento e a promoçẫo do CCF é suportado e orientado pelo modelo ecológico social e pela teoria social cognitiva de Bandura (Resnick, Boltz, Galik, \& Pretzer-Aboff, 2012). Os seus pressupostos centram-se em ajudar as PI a otimizar e a manterem as suas capacidades e a continuarem a aumentar o tempo gasto em AF, para que, desta forma, recuperem e/ou mantenham o mais elevado grau de funcionalidade e previnam o descondicionamento físico e cognitivo. Por fim, baseia-se nas interaçóes entre o cuidador e as PI. As autoras Resnick et al. (2012) sugerem a utilização de quatro componentes na sua implementação (Tabela 1; Resnick \& Boltz, 2019). O essencial é que esta abordagem seja a base de todas as interaçôes e atividades inerentes ao cuidado às PI. Estas são iniciadas sequencialmente, contudo as intervençôes começadas dentro de cada componente são continuamente utilizadas até se tornarem rotinas de cuidado previstas na organização (Resnick et al., 2012). Os estudos de eficácia demonstraram que a implementação do CCF alterou o ambiente hospitalar e as políticas que facilitam a AF (Burket et al., 2013; Resnick et al., 2016), aumentou o CCF durante a hospitalização (Boltz, Chippendale, Resnick, \& Galvin, 2015; Resnick et al., 2016) e no pós-alta melhorou a funcionalidade, aumentou a AF e reduziu os reinternamentos (Boltz et al., 2015; Resnick et al., 2016).

\section{Questáo de investigaçáo/Hipóteses}

Qual é a eficácia da implementação da filosofia do CCF em serviços de medicina interna na promoção da funcionalidade pelos enfermeiros e na prevenção do DF das PIH? Hipótese 1: Existem diferenças estatisticamente significativas com a implementação do CCF na promoção da funcionalidade pelos enfermeiros entre o grupo experimental e o grupo de controlo.

Hipótese 2: Existem diferenças estatisticamente significativas com a implementação do CCF e o DF entre o grupo experimental e o grupo de controlo.

\section{Metodologia}

Realizou-se um estudo quase-experimental prospetivo em quatros serviços de medicina interna (SMI). O grupo de intervenção (experimental) e o grupo de controlo foram selecionados aleatoriamente (um serviço de internamento de homens e um de mulheres em cada grupo), por um investigador externo ao estudo, usando um gerador de números aleatórios. $\mathrm{O}$ estudo foi aprovado pela Comissão de Ética do hospital, com parecer n. ${ }^{\circ}$ CHUC-065-14. A participação foi voluntária e todos os participantes assinaram o consentimento informado livre e esclarecido. A população foi constituída por PIH com 70 e mais anos. A amostragem foi não-probabilística, consecutiva. Foi calculado o tamanho da amostra através do software G-power, que correspondeu a 102 participantes. Foram incluídos os utentes com 70 e mais anos, com capacidade para compreender e interpretar as questôes do questionário (na sua impossibilidade solicitou-se ao cuidador informal) e que aceitassem participar no estudo. Foram excluídas as PHI transferidas de unidades de cuidados intensivos, com doença terminal ou neurodegenerativa, totalmente dependentes na baseline ou com internamentos inferiores a 48 horas. No momento da admissão nos SMI, 117 utentes preenchiam critérios de elegibilidade: 52 no grupo de controlo e 65 no grupo de experimental. Na Figura 1 são representados os procedimentos de amostragem e as razóes dos drop-outs. No final, a amostra foi constituída por 101 utentes. 


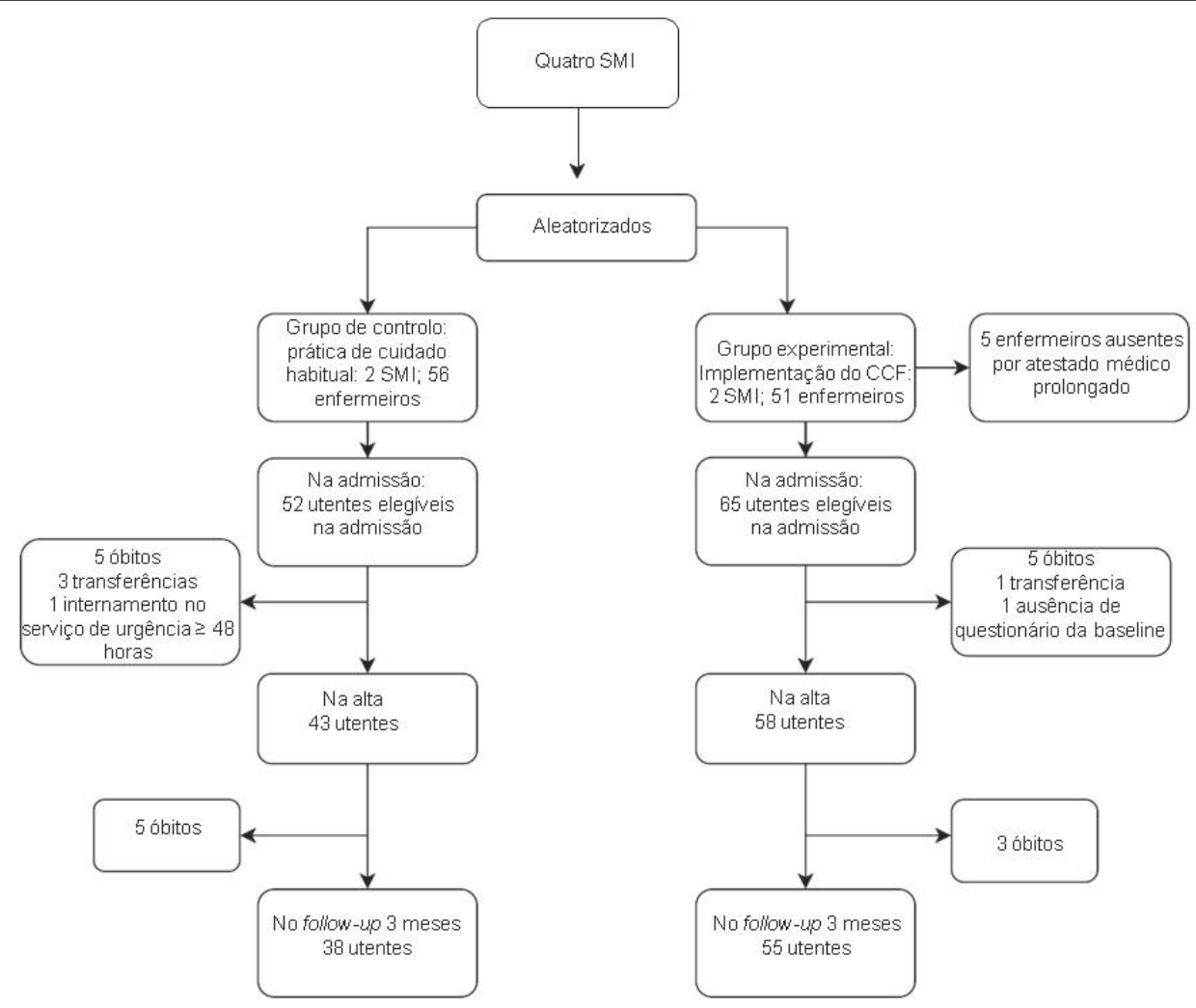

Figura 1. Diagrama do processo de seleção da amostra.

Nota. SMI - Serviço de Medicina Interna; CCF - Cuidado Centrado na Funcionalidade

A implementação da filosofia do CCF foi coordenada pelos investigadores (JT, JG e LN), em parceria com os enfermeiros chefes das unidades experimentais e em articulação com o Serviço de Formação e Aperfeiçoamento Profissional do Hospital. A implementação do CCF decorreu entre março e julho de 2016, tendo sido implementadas as quatro componentes do CCF (Tabela 1). Em cada unidade do grupo experimental foi solicitada a colaboração de um enfermeiro especialista em reabilitação, denominado de Champion. Estes enfermeiros constituíram o elo entre os investigadores e as respetivas equipas de enfermagem, sendo essenciais na implemen- tação do CCF, na manutenção da equipa focada e entusiasmada e na identificação de participantes elegíveis para o estudo. Antes da recolha de dados, as primeiras duas componentes foram implementadas, num período de dois meses (março e abril), para promover mudanças nos serviços e na prática do cuidado dos enfermeiros. Após este período, os investigadores, em parceira com os Champions, continuaram no grupo experimental a motivar as equipas para promover o CCF. Nas enfermarias de controlo foi apenas realizada avaliaçáo (componente I). Nestas unidades, os enfermeiros mantiveram a sua prática habitual de cuidado.

Tabela 1

Descrição das componentes e atividades do CCF em contexto hospitalar

\begin{tabular}{|c|c|}
\hline Componentes & Descrição e atividades \\
\hline $\begin{array}{l}\text { I - Avaliaçáo do } \\
\text { ambiente e das políticas }\end{array}$ & $\begin{array}{l}\text { Foram realizadas } 4 \text { visitas às unidades e entrevistados os enfermeiros(as) chefes para avaliar o } \\
\text { ambiente (18 itens) e as políticas ( } 15 \text { itens), em que mais de metade dos critérios avaliados neces- } \\
\text { sitam de desenvolvimento no sentido de integrar o CCF (Tavares, Grácio, \& Nunes, 2017). Nas } \\
\text { unidades experimentais, foram realizadas algumas alteraçóes para otimizar funcionalidade/AF (ex.: } \\
\text { dispositivos de orientação, corredores desimpedidos). }\end{array}$ \\
\hline $\begin{array}{l}\text { II - Educaçáo da equipa } \\
\text { sobre o CCF }\end{array}$ & $\begin{array}{l}\text { O programa foi desenvolvido segundo as orientaçóes de Resnick et al. (2012) para o CCF em } \\
\text { contexto hospitalar. } \\
\text { O programa decorreu no mês de abril de 2016, em } 4 \text { workshops para cada enfermaria do grupo } \\
\text { de experimental, num total de } 8 \text { sessóes formativas ( } 5 \mathrm{~h} / \text { sessão), nas quais participaram todos os } \\
\text { enfermeiros dessas unidades }(n=51 \text { ), exceto os ausentes por baixa médica prolongada. A formaçáo } \\
\text { foi avaliada relativamente a: autoavaliaçáo do conhecimento prévio; conhecimento após a partici- } \\
\text { paçáo no programa formativo e aplicabilidade para a prática clínica. }\end{array}$ \\
\hline
\end{tabular}




\begin{tabular}{|c|c|}
\hline $\begin{array}{l}\text { III - Estabelecimento } \\
\text { de metas/objetivos }\end{array}$ & $\begin{array}{l}\text { Através dos dados da avaliação inicial, os enfermeiros foram motivados a estabelecer objetivos } \\
\text { a curto e a médio prazo para os utentes. A incorporação do CCF no processo de enfermagem } \\
\text { incluiu: planeamento do cuidado (prescrição das intervençôes), sendo que os objetivos devem ser } \\
\text { baseados nas capacidades das PI, com um propósito para esta, fundamentados na comunicação } \\
\text { entre as PI, a família e os profissionais, e trabalhando com todos os elementos da equipa. }\end{array}$ \\
\hline $\begin{array}{l}\text { IV - Motivação } \\
\text { contínua e mentoria } \\
\text { das equipas, utentes e } \\
\text { famílias }\end{array}$ & $\begin{array}{l}\text { Durante a intervenção, a equipa de investigação e os Champions motivaram e tutoriaram as equi- } \\
\text { pas para incorporar o CCF no desenvolvimento do cuidado através de: estratégias de autoeficácia } \\
\text { para motivar a equipa e os utentes; supervisão e avaliaçáo com feedback positivo quando incorpo- } \\
\text { rado o CCF; material pedagógico para os utentes e famílias sobre o CCF; um programa de manu- } \\
\text { tenção formativo às equipas, com duraçáo de } 18 \text { semanas (incluiu o envio semanal de estratégias } \\
\text { de incorporação do CCF e informaçáo baseada na evidência; material de bolso (pocket cards) para } \\
\text { que os enfermeiros pudessem fazer uma consulta rápida quando se encontrassem em funções nos } \\
\text { serviços; a afixação de material de incentivo à funcionalidade e AF, em locais visíveis da enfermaria } \\
\text { e na sala de reunióes da equipa médica, com o objetivo de lembrar não só os profissionais de saúde } \\
\text { da importância da temática, como também os próprios utentes e respetivas famílias. }\end{array}$ \\
\hline
\end{tabular}

Nota . CCF = Cuidado Centrado na Funcionalidade; $\mathrm{AF}=$ Atividade Física; $\mathrm{PI}=$ Pessoa idosa.

Do protocolo de avaliação constava informação descritiva no momento da admissão, tais como, variáveis sociodemográficas (ex.: idade, sexo, escolaridade, estado civil), clínicas (ex.: diagnóstico de admissão, número de medicamentos, depressão (pergunta dicotómica de item único), perda ponderal (um ou mais quilos nos últimos três meses) e dor, condiçôes geriátricas (ex.: delirium - Método de Avaliação da Confusão, status cognitivo - Teste de Declínio Cognitivo de 6 Itens, medo de cair - pergunta dicotómica de item único, risco de queda escala de Morse, risco de desenvolvimento de lesão por pressão - Escala de Braden, Índice de Comorbilidade de Charlson (ICC), o Risco Relativo Estimado de Morte RREM, ocorrência de queda e funcionalidade (através do Índice de Katz - IK)). Na alta foi avaliada a duração do internamento, o local de residência após a alta e a funcionalidade. No follow-up, foi avaliada a funcionalidade. As características dos enfermeiros foram recolhidas mediante questionário às equipas de enfermagem e inclui variáveis socioprofissionais (ex.: sexo, idade, anos de experiência profissional e no serviço). As horas de cuidados (por enfermeiro e enfermaria) foram recolhidas através do Sistema de Classificação de Doentes (SCD).

Para avaliar a promoção da funcionalidade foi utilizada a Checklist do Cuidado Centrado na Funcionalidade (CC$\mathrm{CF}$ ), desenvolvida por Resnick e colaboradores (Resnick, Rogers, Galik, \& Gruber-Baldini, 2007). Esta escala inclui 19 atividades decorrentes da interação entre o enfermeiro e a pessoa idosa que promovem a funcionalidade, tais como mobilidade na cama, transferências, lavar e vestir a parte superior e inferior do corpo; uso de dispositivos de apoio; andar e movimentos articulares.

A checklist é pontuada através do cálculo do número de atividades promotoras da funcionalidade, realizadas pelos profissionais de saúde, dividido pelo número total de atividades observadas (ex.: 5/10 ou 50\%, indica que foram realizadas metade de todas as atividades possíveis promotoras da funcionalidade). Quanto mais elevada a percentagem mais atividades promotoras da funcionalidade foram realizadas. A versão portuguesa da CCCF mostrou elevados níveis de índice de validade de conteúdo, probabilidade de chance e Kappa modificado, o que suporta a validade de conteúdo deste instrumento (Tavares, Grácio, \& Nunes, 2016).

Para determinar o outcome declínio funcional, utilizou-se o índice de Katz (Duque, Gruner, Clara, Ermida, e Veríssimo, n.d.) que inclui seis atividades básicas de vida diária (ABVD), em que cada uma pontua com 0 (dependente) ou 1 (independente).

O DF foi avaliado através da diferença entre os scores do IK em três intervalos: t0 (diferença entre a baseline e a alta), t1 (entre a alta e o follow-up de três meses) e t2 (entre a baseline e o follow-up), sendo operacionalizado com perda de pelo menos um ponto entre avaliaçóes. O processo de colheita de dados decorreu de 1 de maio a 7 de outubro de 2016: a baseline, o internamento e a alta, de 1 de maio a 30 de junho; e o follow-up, de 5 de agosto a 7 de outubro. Para a identificação dos utentes utilizou-se a informação dos Champions ou enfermeiros dos SMI e o processo informático para sinalizar os utentes que tinham sido admitidos nas últimas 48 horas. Após verificação dos critérios de inclusão e exclusão, as PIH eram convidadas a participar. Nas primeiras 48 horas, após o consentimento informado ser assinado, iniciava-se a recolha dos dados. Para obtenção de informação, recorreu-se preferencialmente ao utente ou, na sua impossibilidade, solicitou-se informação junto dos cuidadores informais, da equipa de saúde (nomeadamente enfermeiros, médicos e assistentes operacionais), através da consulta do diário clínico e do processo clínico eletrónico.

Os investigadores realizaram a recolha de informação nos três momentos através de heteropreenchimento do questionário. A caracterização descritiva e os dados da funcionalidade na baseline foram recolhidos nas primeiras 48 horas. $\mathrm{Na}$ alta, os investigadores avaliaram a funcionalidade junto das PIH. Contudo, alguns utentes tiveram alta em dias em que os investigadores não se encontravam no serviço, tendo-se utilizado o contacto telefónico. No follow-up foi utilizada a entrevista telefónica e recorreu-se preferencialmente ao utente e/ou cuidadores (in)formais que participaram nos momentos anteriores.

O preenchimento da CCCF foi realizado pelos investigadores (JT, JG, LN) através da observação direta não participante dos enfermeiros que tinham distribuídos utentes incluídos no estudo ou daqueles a quem haviam sido delegados cuidados de enfermagem. $\mathrm{O}$ preenchimento da CCCF decorreu entre o terceiro e o quinto dia de internamento (tendo como referência a média de internamento nos SMI, calculada anteriormente com base em dados fornecidos pelo hospital), tendo sido realizada uma observação da interação 
enfermeiro-utente do estudo. A observaçáo decorreu, preferencialmente, no turno da manhá, sendo o tempo mínimo de observação de 30 minutos. A observação, através da checklist, teve por base o protocolo elaborado pelas autoras originais, traduzido pelos investigadores, o que minimiza os vieses decorrentes da observação direta por investigadores diferentes e aumenta a fiabilidade dos resultados.

Para a análise dos dados utilizaram-se técnicas de estatística descritiva e de estatística inferencial. Na estatística descritiva utilizaram-se, para as variáveis contínuas, medidas de tendência central como a média, a mediana e os quartis; e medidas de dispersão como o desvio-padrão; e nas variáveis nominais as frequências relativas (percentagens). Nas análises comparativas utilizaram-se: o teste $t$ de Student (quando a normalidade da distribuição não se verificou usou-se o teste $U$ de Mann-Whitney); a ANOVA (quando a normalidade da distribuição não se verificou usou-se o teste Kruskal-Wallis); o Qui-quadrado (quando os pressupostos do Qui-quadrado não se verificaram usou-se o teste exato de Fisher) e teste de correlação (coeficiente de correlaçáo de Pearson ou Spearman). No tratamento estatístico dos dados foi utilizado o programa estatístico IBM SPSS Statistics, versão 23.0. Os dados das pessoas que faleceram durante o internamento ou dentro do período de follow-up foram excluídos da análise. Foi considerado como estatisticamente significativo o valor de $p<0,05$.

\section{Resultados}

\section{Descriçáo da amostra}

Seguidamente apresentam-se as características do grupo experimental versus (vs) grupo de controlo. A maioria é do sexo feminino ( $50 \%$ vs $58,1 \%$ ), a média de idades é de $81,91 \pm 6,57$ vs $83,21 \pm 6,04$ anos. O estado civil mais frequente é viúvo(a) $(49,1 \%$ vs $49,1 \%)$. A escolaridade é baixa $(52,2 \%$ vs $60,5 \%$ - 0 e 2 anos de frequência escolar). A maioria reside em casa de familiares ( $87,9 \%$ vs $76,6 \%)$. Os diagnósticos de admissão mais identificados foram infeçóes ( $44,2 \%$ vs $39,5 \%$ ), seguidos da patologia cardiovascular ( $15,5 \%$ vs $20,9 \%)$. A maioria dos utentes era polimedicada (6,5 vs 7 fármacos). O ICC (0-8) teve uma mediana de $6(4-7)$ e o RREM $(0-19,37)$ de 9,23 $(4,4-13,4)$ nos dois grupos. A perda ponderal $(59,9 \%$ vs $43,1 \%$ ), o risco elevado de queda ( $48,8 \%$ vs $47,4 \%)$, o medo de cair $(61,1 \%$ vs $55 \%$ ), o risco de lesão por pressão elevado (32,6\% vs $32,1 \%$ ), o declínio cognitivo $(38,6 \%$ vs $46,5 \%)$ e sentir-se triste ou deprimido frequentemente $(70,2 \%$ vs $65,1 \%)$ foram muito similares. Não se verificaram diferenças estatisticamente significativas destas variáveis e o grupo de experimental e controlo, o que significa que os grupos são semelhantes.

As equipas de enfermagem no grupo experimental e de controlo são maioritariamente do sexo feminino $(74 \%$ vs $82 \%)$. A média de idade é $37,5 \pm 8,3$ vs $36,6 \pm 8$ anos e a experiência profissional no serviço é de 74 vs 82 meses. A maioria trabalha $40 \mathrm{~h} /$ semanais $(53,5 \%$ vs $57,3 \%)$ e tem um contrato individual de trabalho (50\% vs $60 \%)$. Não se verificaram diferenças estatisticamente significativas entre os grupos, quanto a estas variáveis.

\section{Cuidado centrado na funcionalidade}

A promoçáo do CCF apresenta uma média de 0,46 \pm 0,22 , o que indica que só $46 \%$ de todas as atividades possíveis foram realizadas com base na promoção da funcionalidade. No grupo de controlo, a média foi de $0,39 \pm 0,19$ e no grupo experimental foi de $0,52 \pm 0,24$, cuja diferença tem significância estatística $(t(91)=-2,85$; $p=0,01) . \mathrm{Na}$ análise por enfermaria, identificaram-se diferenças estatisticamente significativas entre os grupos $(F=5,49 ; p<0,01)$ : enfermarias 1 (controlo) e 4 (experimental; $p<0,01$ ), náo se verificando outras diferenças entre os serviços.

Os valores mais elevados de CCF verificaram-se no grupo de pessoas idosas com medo de cair $(p<0,01)$, com contenção $(p=0,02)$, com perda ponderal $(p=0,04) \mathrm{e}$ depressão $(p=0,04)$ e mais dependentes no IK da baseline $\left(r_{\mathrm{s}}=-0,3 ; p=0,04\right)$. Não existe efeito da interação entre estas variáveis e os grupos na promoção do CCF $(p \geq 0,05)$. Não se verificaram correlaçóes significativas nos valores de promoçáa do CCF e a idade das pessoas idosas $(p=0,13)$, o índice de comorbidade $(p=0,08)$, o número de medicamentos $(p=0,19)$, o IK na alta $(p$ $=0,10)$, o IK no follow-up $(p=0,11)$, a intensidade da dor $(p=0,79)$ e o número de horas de cuidados por enfermaria $(p=0,48)$, o número de horas de cuidados por enfermeiro $(p=0,32)$ e a duraçáo do internamento $(p=0,28)$. Igualmente, não se verificaram diferenças estatisticamente significativas nos valores de promoção do CCF e o sexo $(p=0,95)$, a ocorrência de quedas ( $p=$ $0,28)$, a presença de défice cognitivo $(p=0,53)$, a escala de Braden $(p=0,71)$, a escala de Morse $(p=0,09)$ e a residência após a alta $(p=0,54)$.

\section{Declínio funcional}

Os grupos sem declínio apresentaram valores mais elevados de CCF (Tabela 2). Contudo, observou-se que não existem diferenças estatisticamente significativas entre a promoção do CCF e o declínio em t0 $(U=30,5 ; p=$ $0,15)$, em t1 $(t(38,82)=6,293 ; p<0,15)$ e em $\mathrm{t} 2(\mathrm{t}(83)$ $=2,49 ; p=0,44)$. Não existe efeito da interação entre o CCF e os grupos no DF $(p \geq 0,05)$. 
Tabela 2

Análise do CCF por variáveis

\begin{tabular}{|c|c|c|}
\hline Variáveis & $\overline{\mathrm{X}}( \pm D P) / M e\left(1^{\circ}\right.$ e $\left.3^{\circ} \mathrm{Q}\right)$ & Valor de $p$ \\
\hline $\begin{array}{l}\text { Experimental } \\
\text { Controlo }\end{array}$ & $\begin{array}{l}0,52(0,24) \\
0,39(0,19) \\
\end{array}$ & $<0,01^{\mathrm{F}}$ \\
\hline $\begin{array}{c}\text { Enfermaria } \\
1 \\
2 \\
3 \\
4 \\
\end{array}$ & $\begin{array}{l}0,32( \pm 0,18) \\
0,45( \pm 0,18) \\
0,58( \pm 0,20) \\
0,58( \pm 0,26)\end{array}$ & $<0,01^{\$}$ \\
\hline $\begin{array}{c}\text { Perda ponderal } \\
\text { Sim } \\
\text { Não }\end{array}$ & $\begin{array}{l}0,46(0,20) \\
0,39(0,26)\end{array}$ & $0,04^{\mathrm{F}}$ \\
\hline $\begin{array}{c}\text { Medo de cair } \\
\text { Sim } \\
\text { Não }\end{array}$ & $\begin{array}{l}0,52(0,18) \\
0,37(0,25)\end{array}$ & $<0,01^{\mathrm{F}}$ \\
\hline $\begin{array}{c}\text { Contençáo } \\
\text { Sim } \\
\text { Não } \\
\end{array}$ & $\begin{array}{l}0,53(0,19) \\
0,42(0,24)\end{array}$ & $0,02^{\mathrm{F}}$ \\
\hline $\begin{array}{c}\text { Depressão } \\
\text { Sim } \\
\text { Não } \\
\end{array}$ & $\begin{array}{c}0,5(0,2) \\
0,39(0,26)\end{array}$ & $0,04^{\mathrm{F}}$ \\
\hline $\begin{array}{c}\text { Declínio em t0 } \\
\text { Ausente } \\
\text { Presente }\end{array}$ & $\begin{array}{l}51(49-61) \\
42(43-83)\end{array}$ & $0,15^{\circ}$ \\
\hline $\begin{array}{c}\text { Declínio em t1 } \\
\text { Ausente } \\
\text { Presente }\end{array}$ & $\begin{array}{l}0,52( \pm 0,15) \\
0,45( \pm 0,24)\end{array}$ & $0,15^{\mathrm{F}}$ \\
\hline $\begin{array}{c}\text { Declínio em t2 } \\
\text { Ausente } \\
\text { Presente }\end{array}$ & $\begin{array}{l}0,45( \pm 0,25) \\
0,48( \pm 0,19)\end{array}$ & $0,44^{\mp}$ \\
\hline
\end{tabular}

Nota. $\overline{\mathrm{X}}=$ Média; $D P=$ Desvio-Padrão; $M e=$ Mediana; $\mathrm{Q}=$ Quartil; $\mathrm{a}=$ Teste U de Mann-Whitney; $\mp=$ Teste $t$ de student; ${ }^{\$}=$ One-way ANOVA.

\section{Discussáo}

A promoção do CCF foi baixa quando comparada com os estudos de Boltz, Capezuti, e Shabbat (2011) e Boltz et al. (2012), $46 \%$ vs $92 \%$ e $76 \%$, respetivamente). Adicionalmente, o estudo original dicotomizou o CCF (CCF $=1$ ou $\mathrm{CCF}<1)$ pela falta de normalidade da variável. Esta dicotomização foi possível pela assimetria positiva, isto é, a amostra foi dividida em dois grupos: o que promove o CCF a $100 \%$ e o que promove menos de $100 \%$, o que não se verificou neste estudo. A promoção do CCF, que difere significativamente nos dois estudos, limita a comparaçáo dos resultados. Por outro lado, o CCF como filosofia de cuidado, pode estar incorporada na prática habitual dos enfermeiros norte-americanos, ao contrário da realidade portuguesa.

Resnick e Boltz (2019) identificaram que o CCF é promovido nas pessoas idosas mais jovens $(p=0,028)$. Esta situação não se verificou em Portugal. Uma possível explicação pode dever-se ao facto de a idade média dos utentes ser superior à do estudo original $(82,47 \pm 6,57$ vs $80,8 \pm 7,2$ ), bem como ao facto de nos critérios de inclusão deste estudo se ter optado por idades iguais ou superiores a 70 anos. Por outro lado, este estudo decorreu em SMI, cuja média de idades é elevada.

As características intrapessoais dos enfermeiros também poderâo ter sido determinantes na predisposição para práticas menos consistentes com o CCF, nomeadamente as características sociodemográficas, conhecimentos e crenças (Resnick \& Boltz, 2019). Estudos demonstram uma diferença significativa entre o ambiente de trabalho dos enfermeiros e os resultados de saúde para os utentes, enfermeiros e organizaçôes (Aiken et al., 2018). Investigaçôes realizadas em Portugal demonstraram que o ambiente geriátrico de trabalho dos enfermeiros é desfavorável: existem barreiras à promoção de cuidado de enfermagem de qualidade, pouco conhecimento e atitudes negativas destes profissionais (Tavares, Silva, Sá-Couto, Boltz, \& Capezuti, 2017), fatores que contribuem para a não incorporação do CCF.

$\mathrm{O}$ desenvolvimento das atividades promotoras do CCF não se encontra associado às horas de cuidados, o que reforça a ideia central desta filosofia ao defender que a realização destas atividades não implica um incremento de tempo dedicado ao cuidado. Ressalva-se, contudo, que o SCD pode não refletir a complexidade e especificidade do cuidado à pessoa idosa. Devem, por isso, realizar-se estudos que procurem analisar o impacto dessas características na perceção do ambiente de trabalho dos enfermeiros, na perceção da sobrecarga e nos resultados em saúde.

Verificou-se no presente estudo que as atividades de CCF foram mais realizadas nas pessoas idosas mais dependentes na admissão, mais deprimidas, com medo de cair, com perda ponderal e contidas. Este estudo alude a uma problemática no desenvolvimento do cuidado às pessoas idosas independentes. Verificou-se que o DF é mais acentuado 
neste grupo (54,4\% totalmente independentes na admissão vs $30,7 \%$ na alta; Tavares et al., 2018). A associação significativa negativa, embora fraca, sugere que, quanto mais independentes são as PI, menos atividades promotoras da independência são realizadas, o que as pode colocar em maior risco de declínio funcional.

Contrariamente aos estudos que analisaram o CCF (Boltz et al., 2011, 2012; Resnick et al., 2016), observou-se que não existem diferenças estatisticamente significativas entre a prática do CCF e o DF. Contudo, verificou-se que as pessoas idosas sem declínio foram aquelas que mais beneficiaram do CCF. Importa considerar que a prática do CCF é manifestamente inferior à verificada nos estudos sobre esta filosofia, o que pode limitar o seu impacto na prevenção do declínio. Acresce a necessidade de implementar, de forma mais consistente as 4 componentes de implementação do CCF para que este cuidado possa ser incorporado na prática dos enfermeiros. Importa destacar, que no processo de implementação, a componente I não demonstrou ser favorecedora da incorporação do CCF (Tavares, Grácio, $\&$ Nunes, 2017). Adicionalmente, verificou-se uma baixa adesão na componente III (estabelecimento de objetivos) e pouca clareza do papel do champion enquanto elemento motivador das equipas (componente IV).

Almeja-se, assim, uma mudança mais eficaz ao nível do ambiente físico e das políticas organizacionais, com a implementaçấo de normas institucionais, estratégias educativas que envolvam não só os enfermeiros como os outros elementos da equipa de saúde, nomeadamente os assistentes operacionais, familiares e cuidadores. Deve proceder-se igualmente ao estabelecimento de metas funcionais baseadas na avaliação multidimensional e numa perspetiva de co-design com os utentes, assim como a um trabalho interdisciplinar e ao desenvolvimento de estratégias de motivação e reforço positivo para todos os intervenientes no CCF. É basilar priorizar este cuidado nos hospitais, criando ambientes de trabalho favoráveis à prática de enfermagem, para que o CCF possa ter uma expressão significativa na funcionalidade $\mathrm{e}$ na qualidade de vida das pessoas idosas hospitalizadas.

Relativamente às limitaçóes deste estudo, em primeiro, destaca-se o tamanho pequeno da amostra dos grupos e o contexto, que inclui apenas 4 SMI num centro hospitalar e universitário localizado na região centro do país. Estes fatores limitam a generalização dos resultados. $\mathrm{O}$ desenvolvimento de estudos aleatorizados controlados, com amostras maiores, a inclusão de outros serviços (quer de especialidades médicas ou cirúrgicas) e de diferentes tipos de hospitais em outras regióes do país, são fatores que podem ajudar a explorar o impacto da implementaçáo do CCF em contexto hospitalar.

Em segundo lugar, a observação do CCF foi deliberadamente realizada no período da manhã de forma a contemplar um maior número de ABVD. É possível, no entanto, que estas atividades possam ter ocorrido noutros períodos e que tenham sido descritas como não observadas. Pesquisas futuras deverẫo considerar a realização de observaçóes diretas durante períodos diferentes de tempo ao longo do dia e mais prolongados. No que concerne à observação, importa referir o efeito da desejabilidade social que pode enviesar os parâmetros avaliados, ainda que tenham sido utilizadas técnicas de minimização, como observaçôes não programadas. Uma observação prévia à implementação do estudo seria relevante para: conhecer a baseline do CCF; analisar a predisposição das equipas de enfermagem em relação à funcionalidade; e analisar o impacto do programa educativo nas equipas. Este estudo excluiu os utentes totalmente dependentes. Contudo, seria pertinente observar neste grupo de utentes a prática do CCF e comparar com o grupo de utentes com menor dependência, uma vez que neste estudo se verificou que as atividades promotoras da funcionalidade aconteciam em maior número nos utentes com graus de dependência mais elevados.

Em terceiro, alguns fatores relacionados com o ambiente de trabalho dos enfermeiros e políticas organizacionais podem ter condicionado o envolvimento na filosofia do CCF. Estudos demonstram que as perceçóes negativas em relação ao suporte organizacional, que promove a autonomia e o controlo dos enfermeiros na resoluçáo de problemas, afetam a qualidade do cuidado (Tavares et al., 2017). Pesquisas futuras deverão incorporar uma avaliação mais ampla acerca das características intrapessoais dos enfermeiros e respetivas repercussôes no cuidado à PI. Também seria pertinente considerar o nível de (i) mobilidade dos utentes no hospital, o que iria alargar a compreensão do impacto do repouso no leito e das práticas do CCF nas PIH em Portugal.

Por último, considera-se que a avaliação da satisfação dos utentes, familiares/cuidadores e profissionais de saúde é relevante para assegurar a fiabilidade da prática do CCF. Futuros estudos deverão igualmente considerar uma componente de avaliação qualitativa e a inclusão do papel desempenhado pela família/cuidador na promoção do CCF durante e após o internamento hospitalar das PI.

\section{Conclusáo}

Este estudo foi pioneiro na implementação do CCF em Portugal, não existindo estudos similares. Os resultados demonstram que apenas $46 \%$ de todas as atividades possíveis foram realizadas com base na promoção funcional. A implementação do CCF demonstrou uma diferença estatisticamente significativa entre os dois grupos, sendo mais favorável no grupo experimental. Contudo, este efeito não se verificou na prevenção do DF. A promoção da funcionalidade através do CĆF é perspetivada como um desafio e uma oportunidade de mudança, inovação e criatividade, no sentido de melhorar a eficiência e a qualidade do cuidado às PIH, levando à implementação de iniciativas integradas e continuadas para responder às necessidades destes utentes.

\section{Contribuiçáo de autores}

Conceptualização: Tavares, J. P., Grácio, J., Nunes, L. V. Metodologia: Tavares, J. P., Grácio, J., Nunes, L. V. Investigação: Tavares, J. P., Grácio, J., Nunes, L. V. Tratamento de dados: Tavares, J. P., Grácio, J., Nunes, L. V. 
Análise formal: Tavares, J. P., Grácio, J., Nunes, L. V. Redação - rascunho original: Tavares, J. P.

Redação - análise e edição: Tavares, J. P., Grácio, J., Nunes, L. V

\section{Agradecimentos}

Agradecemos à Direção de Enfermagem do Centro Hospitalar e Universitário de Coimbra, nas pessoas do Excelentíssimo Senhor Enfermeiro Diretor António Marques, à data, e da Excelentíssima Senhora Enfermeira Supervisora Eugénia Morais, pelo apoio no desenvolvimento deste estudo.

\section{Financiamento}

Centro de Investigação em Tecnologias e Serviços de Saúde (CINTESIS), referência UIDB/4255/2020.

\section{Referências bibliográficas}

Admi, H., Shadmi, E., Baruch, H., \& Zisberg, A. (2015). From research to reality: Minimizing the effects of hospitalization on older adults. Rambam Maimonides Medical Journal, 6(2), e0017. doi:10.5041/RMMJ.10201

Aiken, L. H., Cerón, C., Simonetti, M., Lake, E. T., Galiano, A., Garbarini, A., ... Smith, H. L. (2018). Hospital nurse staffing and patient outcomes. Revista Médica Clinica Las Condes, 29(3), 322-327. doi:10.1016/J.RMCLC.2018.04.011

Boltz, M., Capezuti, E., \& Shabbat, N. (2011). Nursing staff perceptions of physical function in hospitalized older adults. Applied Nursing Research, 24(4), 215-22 doi:10.1016/j.apnr.2010.01.001

Boltz, M., Chippendale, T., Resnick, B., \& Galvin, J. E. (2015). Testing family-centered, function-focused care in hospitalized persons with dementia. Neurodegenerative Disease Management, 5(3), 203-215. doi:10.2217/nmt.15.10

Boltz, M., Resnick, B., Capezuti, E., Shuluk, J., \& Secic, M. (2012). Functional decline in hospitalized older adults: Can nursing make a difference? Geriatric Nursing, 33(4), 272-279. doi:10.1016/j. gerinurse.2012.01.008

Brown, C. J., Redden, D. T., Flood, K. L., \& Allman, R. M. (2009). The underrecognized epidemic of low mobility during hospitalization of older adults. Journal of the American Geriatrics Society, 57(9), 1660-1665. doi:10.1111/j.1532-5415.2009.02393.x

Burket, T. L., Hippensteel, D., Penrod, J., \& Resnick, B. (2013). Pilot testing of the function focused care intervention on an acute care trauma unit. Geriatric Nursing, 34(3), 241-246. doi:10.1016/j. gerinurse.2013.02.001
Dahlke, S. A., Hunter, K. F., \& Negrin, K. (2019). Nursing practice with hospitalised older people: Safety and harm. International Journal of Older People Nursing, 14, e12220. doi:10.1111/opn.12220

Dermody, G., \& Kovach, C. R. (2018). Barriers to promoting mobility in hospitalized older adults. Research in Gerontological Nursing, 11(1),17-27. doi: 10.3928/19404921-20171023-01

Duque, S., Gruner, H., Clara, J., Ermida, J., \& Veríssimo, M. (n.d.). Avaliação geriátrica. Lisboa, Portugal: Núcleo de Estudos de Geriatria da Sociedade Portuguesa de Medicina Interna. Recuperado de http://www.spmi.pt/docs_nucleos/GERMI_36.pdf

Resnick, B., Boltz, M., Galik, E., \& Pretzer-Aboff, I. (2012). Restorative care nursing for older adults. New York, NY: Springer Publishing Company

Resnick, B., Rogers, V., Galik, E., \& Gruber-Baldini, A. L. (2007). Measuring restorative care provided by nursing assistants: Reliability and validity of the Restorative Care Behavior Checklist. Nursing Research, 56(6), 387-398. doi:10.1097/01. NNR.0000299854.52429.ac

Resnick, B., \& Boltz, M. (2019). Optimizing function and physical activity in hospitalized older adults to prevent functional decline and falls. Clinics in Geriatric Medicine, 35(2), 237-251. doi:10.1016/j.cger.2019.01.003

Resnick, B., Wells, C., Galik, E., Holtzman, L., Zhu, S., Gamertsfelder, E., ... Boltz, M. (2016). Feasibility and efficacy of function-focused care for orthopedic trauma patients. Journal of Trauma Nursing : The Official Journal of the Society of Trauma Nurses, 23(3), 144-155. doi:10.1097/JTN.0000000000000203

Tavares, J., Grácio, J., \& Nunes, L. (2018). Hospitalized older adults: Functional trajectory in Portuguese hospital. Revista de Enfermagem Referência, 4(18), 19-28. doi:10.12707/RIV18028

Tavares, J., Grácio, J., \& Nunes, L. (2017). Avaliação do ambiente e políticas hospitalares: Rumo a um hospital amigo das pessoas idosas. Revista Brasileira de Geriatria e Gerontologia, 20(2), 254258. doi:10.1590/1981-22562017020.160095

Tavares, J., Grácio, J., \& Nunes, L. (2016). Functional focused care: Content validity of functional focused care behavior checklist. European Geriatric Medicine, 7(suppl. 1), S1-S282.

Tavares, J., Silva, A. L., Sá-Couto, P., Boltz, M., \& Capezuti, E. (2017). Percepçáo dos enfermeiros sobre o cuidado a idosos hospitalizados: Estudo comparativo entre as regiôes Norte e Central de Portugal. Revista Latino-Americana de Enfermagem, 25, e2757. doi:10.1590/1518-8345.0839.2757

Zisberg, A., Shadmi, E., Gur-Yaish, N., Tonkikh, O., \& Sinoff, G. (2015). Hospital-associated functional decline: The role of hospitalization processes beyond individual risk factors. Journal of the American Geriatrics Society, 63(1), 55-62 doi:10.1111/jgs.13193 